\title{
Race and socioeconomic disparity in treatment and outcome of traumatic cervical spinal cord injury with fracture: Nationwide Inpatient Sample database, 1998-2009
}

\author{
Alexander B. Dru ${ }^{1} \cdot$ Brett Reichwage $^{1} \cdot$ Dan Neal $^{1} \cdot$ Sasha Vaziri ${ }^{1} \cdot$ Dennis Timothy Lockney $^{1} \cdot$ W. $^{\text {Christopher Fox }}{ }^{1} \cdot$ \\ Brian L. Hoh ${ }^{1} \cdot$ Daniel J. Hoh ${ }^{1}$
}

Received: 5 November 2018 / Revised: 16 March 2019 / Accepted: 3 April 2019 / Published online: 16 April 2019

(c) International Spinal Cord Society 2019

\begin{abstract}
Study design A retrospective national administrative database study.

Objective Advances in treatment of traumatic cervical spinal cord injury with fracture (TCSCIF) have led to significant improvements in clinical outcomes; however, progress in healthcare is seldom ubiquitous across demographic groups. Therefore, we explored if disparities in treatment and outcome after TCSCIF exist across race and socioeconomic status.

Setting USA.

Methods We queried the Nationwide Inpatient Sample database from 1998 to 2009 for TCSCIF hospitalizations. Multivariate analysis was used to identify the correlation between socioeconomic status and race to injury, treatment type, and outcome.

Results There were 21,985 admissions for TCSCIF, $66.9 \%$ of whom had a favorable discharge disposition. In-hospital mortality rate was $12.5 \%$. A total of $43.7 \%$ underwent surgery. Overall, surgery was associated with lower in-hospital mortality (OR $0.30,95 \%$ CI $0.27-0.34, p<0.01$ ) and better discharge disposition (OR 0.68, 95\% CI $0.62-0.74, p<0.01$ ) versus nonsurgical or no intervention. African-American (AA) race and low socioeconomic status (LSES) were significant predictors of lower odds to undergo surgery and unfavorable discharge disposition, respectively; potentially explained by a higher odds of increased New Injury Severity Score classification at presentation. Surgical and favorable discharge rates for LSES and non-Caucasian races, however, have been steadily improving over the study period.

Conclusions Despite trending improved outcomes after TCSCIF, LSES, or AA race were more likely to have worse outcomes compared to their counterparts. In addition, LSES, AA, and Hispanic groups were less likely to undergo surgical treatment, suggesting disparities in management and outcome effect.
\end{abstract}

\section{Introduction}

Understanding and reducing racial disparities in healthcare is a national priority [1]. It is well documented that such inequalities in healthcare exist even when adjusted for

Supplementary information The online version of this article (https:// doi.org/10.1038/s41393-019-0280-6) contains supplementary material, which is available to authorized users.

Alexander B. Dru

Alexander.Dru@neurosurgery.ufl.edu

1 University of Florida, Lillian S. Wells Department of Neurosurgery, Gainesville, FL, USA severity of condition [2, 3]. Traumatic cervical spinal cord injury with fracture (TCSCIF), affects over 11,000 people every year across the USA from which patients acutely present with sensory, motor, and autonomic neurovascular deficits secondary to disruption of the cervical spinal cord [4-6]. In addition, with motor vehicle crashes being the leading cause of TCSCIF over the past 40 years for individuals younger than 45 years old, many of these patients suffer concomitant complex orthopedic, pulmonary, and cardiovascular trauma, necessitating rapid multidisciplinary medical management [7, 8].

There is a continuously growing body of preclinical and clinical data suggesting that decompressive surgery of the spinal cord after injury helps to prevent various secondary mechanisms of injury and helps to improve neurologic outcome [9-12]. In addition, there is a current trend in research 
and practice popularizing early $(<24 \mathrm{~h})$ to ultra-early $(<8 \mathrm{~h})$ surgical decompression and stabilization to regain motor and sensory function in all grades of TCSCIF, including complete injury [13-15]. With timely surgical decompression and stabilization of TCSCIF serving as a mainstay of treatment, inaccessibility to care could limit otherwise potentially recoverable neurologic decline [16, 17].

Advances in treatment of TCSCIF have led to significant improvements in overall clinical outcomes; however, progress in healthcare may not be ubiquitous across demographic groups. If a disparity in surgical treatment of populations exists for an acute traumatic condition, which has proven to benefit from timely intervention, it is the responsibility of healthcare systems to identify and help rectify barriers to optimal treatment. The purpose of this study was to identify potential inequalities in management and outcome for TCSCIF in at-risk populations by analyzing surgical intervention rates, functional outcome, and mortality across various racial and socioeconomic groups.

\section{Methods}

We surveyed the National Inpatient Sample (NIS) database from the Agency for Healthcare Research and Quality, Healthcare Cost and Utilization project. The NIS contains a cohort of hospitalization and discharge data from all hospitals participating in the Healthcare cost and Utilization Project; representing 20\% of all US nonfederal admissions (excluding rehabilitation and long-term acute care facilities). NIS delineates hospitals by region, location, teaching status, size, and ownership. We adhered to methods outlined by NIS protocol to account for these factors in our estimations of the total number of hospitalizations as well as our variables for the years 1998-2009. For further information regarding NIS, please see http://www.hcup-us.ahrq. gov/nisoverview.jsp.

We surveyed the NIS for teaching and nonteaching hospital admissions regarding cervical spine trauma from 1998 to 2009 . To specifically identify hospitalizations in the NIS for cervical trauma where surgery may be indicated, we used the ICD-9 (International Classification of Disease, 9th Revision) diagnosis codes for TCSCIF (806.00-806.09). We excluded diagnosis code '952.0X' (cervical spinal cord injury without evidence of spinal bone fracture). All patients included therefore suffered a traumatic cervical spinal cord injury with associated cervical fracture and a resulting neurological injury.

The first analysis performed was to determine whether intervention type (surgical, nonsurgical, or none) was a predictor of discharge disposition for TSCSIF. This analysis was performed twice, once with discharge disposition as the outcome variable (favorable: home or inpatient rehabilitation hospital, or unfavorable: death, hospice, skilled nursing facility, or long-term acute care hospital, years 1998-2009), and a second time with hospital mortality (died or survived) as the outcome variable. We included age, gender, admission year, hospital bed size, hospital region, and hospital case volume as covariates in all analyses.

The second analysis was to determine a potential association between degree of bodily injury and race or socioeconomic status, as a potential confounding factor for treatment occurrence and outcome. The New Injury Severity Score (NISS) found in NIS is not an equal-interval score variable because patients with 'maximal' damage to any body zone are given the maximal score of 75 , so traditional comparison protocols between race and NISS, such as ANOVA could not be used. Instead, we classified NISS into four categories (minor severity, minor to moderate severity, moderate to major severity, and major severity) based on the quartiles of NISS observed in the dataset: NISS $<13=$ minor, $12<$ NISS $<17=$ minor $/$ moderate, 16 $<$ NISS $<26=$ moderate/major, and NISS $>25=$ major. We then used ordinal logistic regression to determine whether race was a predictor of NISS category. In addition, chi-square test determined whether the socioeconomic status was associated with NISS category.

The third analysis was to explore any potential association between race or socioeconomic status and surgical intervention rates, discharge disposition, and hospital mortality for patients with TCSCIF. Generalized estimating equations (SAS Proc GENMOD) were used to determine whether race (Caucasian, African American, Hispanic, Asian/Pacific Islander, Native American, or Other) was a predictor of unfavorable outcomes for TCSCIF patients. The analysis was performed three times, with surgical intervention, discharge disposition (favorable or unfavorable), and hospital mortality as the outcome variable. The analysis was repeated for low socioeconomic status (LSES), defined by the NIS variable indicating the median income in the patient's ZIP code; with all patients in the lowest ZIP income classified as "low" and all others as "higher," substituted for race. We included age, gender, admission year, hospital bed size, hospital region, hospital case volume, and NISS as covariates in all analyses. Finally, we combined race and socioeconomic status into a single multivariate model (four groups: Caucasian/higher status, Caucasian/ LSES, non-Caucasian/higher status, and non-Caucasian/ LSES) to observe potential association between surgical intervention rates, discharge disposition, and hospital mortality for patients with TCSCIF.

The fourth analysis was to assess any change in trends with respect to surgical intervention, discharge disposition, and mortality rates in minority populations or LSES individuals over time. The proportion of Caucasian patients and the proportion of non-Caucasian patients with TCSCIF 
who had surgical intervention (years 1988-2009), favorable outcomes (years 1998-2009), and mortality (years 1998-2009) were analyzed. Regression analysis was performed for these proportions on race category and year to determine whether race had an effect on the proportion of favorable outcomes. This analysis was repeated with socioeconomic status substituted for race. The SAS statistical software package (version 9.3, SAS Institute Inc., Car, NC, USA) was used to create generalized estimating equations and all linear regression models. All analyzed data can be found in the supplementary materials section.

\section{Results}

The NIS survey resulted in 21,985 admissions for TCSCIF. Patient and hospital characteristics are listed in Table 1. With respect to favorable outcome as a function of hospital intervention, 20,176 patients were queried (observations were not included if discharge disposition information was missing). Patients who received surgical intervention had higher odds of favorable discharge as patients who received no intervention (OR 1.60, 95\% CI 1.46-1.84, $p<0.01$ ). Patients who received nonsurgical intervention had lower odds of favorable discharge as patients who received surgical intervention (OR $0.52,95 \%$ CI $0.42-0.64, p<0.01)$. Patients who received surgical intervention had lower odds of death as patients who received no intervention (OR 0.30, 95\% CI 0.27-0.34, $p<$ 0.01 ), and the odds of survival for patients who received nonsurgical intervention were not significantly different from those who received no intervention $(p=0.44)$.

\section{Effect of race and socioeconomic status on NISS}

With respect to NISS, logistic regression indicated that race was a predictor of NISS category $(p=<0.01)$ for TCSCIF patients. African Americans (OR 1.20, 95\% CI 1.13-1.34, $p<0.01$ ), Hispanics (OR 1.40, 95\% CI 1.28-1.56, $p<$ 0.01 ), Asian/Pacific Islanders (OR 1.30, 95\% CI 1.02-1.55, $p=0.03$ ), Native Americans (OR $1.5095 \%$ CI 1.02$2.12, p=0.04$ ), and 'Other Races' (OR 1.40, 95\% CI 1.131.64, $p<0.01$ ) had higher odds of being in a higher cumulative NISS category compared to Caucasians. Chisquare analysis showed socioeconomic status was not significantly associated with NISS $(p=0.24)$. There were 20,832 observations with information concerning race and/ or socioeconomic status.

\section{Surgical intervention, favorable outcome, and mortality across race and socioeconomic status}

Overall, race was a predictor of surgical intervention $(p=$ 0.04) in TCSCI patients. Both African American (OR 0.83,
Table 1 Patient and hospital demographics, by insurance status. Standard deviation (SD)

\begin{tabular}{|c|c|c|c|}
\hline & $\begin{array}{l}\text { Insured } \\
(n=36,398)\end{array}$ & $\begin{array}{l}\text { Uninsured } \\
(n=8,034)\end{array}$ & $p$-value \\
\hline Age, years $($ mean $\pm S D)$ & $48.4 \pm 22.6$ & $39.0 \pm 15.7$ & $<0.01$ \\
\hline \multicolumn{4}{|l|}{ Race } \\
\hline Caucasian & $18,112(71.8 \%)$ & $3397(61.1 \%)$ & \multirow[t]{6}{*}{$<0.01$} \\
\hline Black & $3867(15.3 \%)$ & $1136(20.4 \%)$ & \\
\hline Hispanic & $1910(7.6 \%)$ & $735(13.2 \%)$ & \\
\hline Asian & $615(2.4 \%)$ & $101(1.8 \%)$ & \\
\hline Native American & $157(0.6 \%)$ & $43(0.8 \%)$ & \\
\hline Other & $576(2.3 \%)$ & $149(2.7 \%)$ & \\
\hline \multicolumn{4}{|l|}{ Socioeconomic status } \\
\hline Low & $8787(25.3 \%)$ & $2357(31.4 \%)$ & \multirow[t]{2}{*}{$<0.01$} \\
\hline Higher & $26,000(74.7 \%)$ & $5154(68.6 \%)$ & \\
\hline \multicolumn{4}{|l|}{ Gender } \\
\hline Female & $10,017(27.5 \%)$ & $1315(16.4 \%)$ & \multirow[t]{2}{*}{$<0.01$} \\
\hline Male & $26,376(72.5 \%)$ & $6716(83.6 \%)$ & \\
\hline \multicolumn{4}{|l|}{ Hospital bed size } \\
\hline Small & $3112(8.6 \%)$ & $419(5.2 \%)$ & \multirow[t]{3}{*}{$<0.01$} \\
\hline Medium & $8428(23.2 \%)$ & $1863(23.3 \%)$ & \\
\hline Large & $24,800(68.2 \%)$ & $5717(71.5 \%)$ & \\
\hline \multicolumn{4}{|l|}{ Hospital teaching status } \\
\hline Nonteaching & $13,613(37.5 \%)$ & $2990(37.4 \%)$ & \multirow[t]{2}{*}{0.89} \\
\hline Teaching & $22,727(62.5 \%)$ & $5009(62.6 \%)$ & \\
\hline \multicolumn{4}{|l|}{ Hospital region } \\
\hline Northeast & $6024(16.5 \%)$ & $830(10.3 \%)$ & \multirow[t]{4}{*}{$<0.01$} \\
\hline Midwest & $8751(24.0 \%)$ & $1597(19.9 \%)$ & \\
\hline South & $12,815(35.2 \%)$ & $3710(46.1 \%)$ & \\
\hline West & $8837(24.3 \%)$ & $1903(23.7 \%)$ & \\
\hline $\begin{array}{l}\text { Annual hospital volume } \\
(\text { mean } \pm \mathrm{SD})\end{array}$ & $22.2 \pm 24.7$ & $23.4 \pm 22.8$ & $<0.01$ \\
\hline $\begin{array}{l}\text { Injury Severity Score } \\
(\text { mean } \pm \text { SD) }\end{array}$ & $21.7 \pm 15.1$ & $22.0 \pm 14.9$ & 0.11 \\
\hline \multicolumn{4}{|l|}{ In-hospital mortality } \\
\hline Died & $3236(8.9 \%)$ & $620(7.8 \%)$ & \multirow[t]{2}{*}{$<0.01$} \\
\hline Survived & $33,071(91.1 \%)$ & $7385(92.3 \%)$ & \\
\hline \multicolumn{4}{|c|}{ Discharge disposition 1 (1998-2009 only) } \\
\hline Favorable & $10,963(71.3 \%)$ & $2746(83.0 \%)$ & \multirow[t]{2}{*}{$<0.01$} \\
\hline Unfavorable & $4412(28.7 \%)$ & $561(17.0 \%)$ & \\
\hline \multicolumn{4}{|c|}{ Discharge disposition 2 (all years) } \\
\hline Routine & $3599(48.6 \%)$ & $11,416(34.7 \%)$ & \multirow[t]{7}{*}{$<0.01$} \\
\hline $\begin{array}{l}\text { Transfer to short-term } \\
\text { hospital }\end{array}$ & $593(8.0 \%)$ & $3020(9.2 \%)$ & \\
\hline $\begin{array}{l}\text { Transfer to another type of } \\
\text { facility }\end{array}$ & $2272(30.7 \%)$ & $13,515(41.1 \%)$ & \\
\hline Home healthcare & $299(4.0 \%)$ & $1808(5.5 \%)$ & \\
\hline Against medical advice & $73(1.0 \%)$ & $159(0.5 \%)$ & \\
\hline Died & $571(7.7 \%)$ & $2966(9.0 \%)$ & \\
\hline $\begin{array}{l}\text { Discharged alive, } \\
\text { destination unknown }\end{array}$ & $0(0 \%)$ & $4(0.01 \%)$ & \\
\hline \multicolumn{4}{|l|}{ Admission source } \\
\hline Transfer & $4664(14.9 \%)$ & $677(9.8 \%)$ & \multirow[t]{2}{*}{$<0.01$} \\
\hline Other & $26,685(85.1 \%)$ & $6268(90.2 \%)$ & \\
\hline
\end{tabular}

95\% CI $0.73-0.94, p<0.01)$ and Hispanic (OR 0.87, 95\% CI $0.76-0.99, p=0.03$ ) TCSCIF patients had lower odds of receiving surgery versus nonsurgical or no intervention compared to Caucasians. There were 14,847 observations in 
the dataset used for analysis; 6795 of those patients received a surgical intervention. LSES TCSCIF patients also had lower odds of receiving surgery versus nonsurgical or no intervention (OR 0.87 , 95\% CI $0.82-0.94, p<0.01$ ) or nonsurgical versus no intervention (OR $0.80,95 \%$ CI $0.67-$ 0.94, $p=0.01)$. There were 20,758 observations in the dataset used for analysis; 9073 of those patients received a surgical intervention.

Overall, minority race (African American, Hispanic, Asian/Pacific Islander, Native American, or 'Other Races') was not significantly different compared to Caucasians with respect to favorable discharge; however, African American TCSCIF patients had lower odds of favorable discharge (OR 0.80, 95\% CI 0.72-0.89, $p<0.01$ ). There were 13,079 observations in the dataset used for analysis; 4002 patients had favorable discharge and 3412 had routine discharge. When LSES was substituted for race in the TCSCIF population, this group had lower odds of favorable discharge (OR 0.87, 95\% CI 0.77-0.98, $p=0.02$ ) and routine discharge (OR 0.88, 95\% CI 0.79-0.97, $p=0.01$ ) compared to higher income status. There were 19,014 observations in the dataset used for analysis; 5492 of those patients had favorable discharge and 5469 had routine discharge. Race and socioeconomic status were not significant predictors of in-hospital mortality.

Our multivariate model that controlled for race and socioeconomic status demonstrated that higher status nonCaucasians had lower odds of receiving surgery than higher status Caucasians (OR 0.89, 95\% CI 0.81-0.97, $p=0.01$ ). LSES non-Caucasians had lower odds of receiving surgery than higher status Caucasians (OR 0.83, 95\% CI 0.73-0.94, $p<0.01)$. Higher status non-Caucasians had lower odds of receiving surgery than LSES Caucasians (OR 0.87, 95\% CI $0.77-0.99, p=0.03)$. LSES non-Caucasians had lower odds of receiving surgery than LSES Caucasians (OR 0.82, 95\% CI $0.71-0.94, p=0.01)$. For favorable discharge status higher status non-Caucasians, LSES non-Caucasians, and LSES Caucasians all had lower odds of favorable discharge compared to higher status Caucasians (OR 0.83, 95\% CI $0.73-0.95, p=0.01 /$ OR $0.69,95 \%$ CI $0.59-0.81, p<0.01 /$ OR $0.75,95 \%$ CI $0.66-0.85, p<0.01)$, respectively. Finally combined race-status was not a significant predictor of inhospital mortality.

\section{Trends in surgical intervention, favorable outcome, and mortality over time for race and socioeconomic status}

We calculated the proportion of Caucasian patients and the proportion of non-Caucasian patients all with TCSCIF who had surgical intervention (1988-2009), favorable outcomes (years 1998-2009), and mortality (years 1998-2009). These metrics were analyzed to determine any potentially deviating trends. With respect to surgical intervention (see Fig. 1), rates were increasing for all races combined $(p<$ 0.01 for effect of year). There was no difference in surgical intervention rate changes for Caucasians and nonCaucasians $(p=0.47)$, African-American patients and Caucasian patients $(p=0.34)$, Hispanic patients and Caucasian patients $(p=0.19)$, or lower and higher socioeconomic status patients $(p=0.75)$.

Figure 2 illustrates the trends in favorable discharge rate for various races and socioeconomic status compared to that of Caucasian patients and high-income classes, respectively. Favorable outcome rates were improving for all races $(p<$ 0.01 for effect of year), improving for both African Americans and Caucasians ( $p=0.05$ for effect of year), but no evidence that they were improving more rapidly for one race versus the other $(p=0.42)$. In addition, favorable outcome rates improved for Hispanics $(p<0.01$ for effect of year), and improved more rapidly for Hispanics than for Caucasians $(p=0.02)$. With respect to socioeconomic class, favorable outcome rates improved for all classes $(p=0.03$ for effect of year), but not more rapidly for patients of higher status than for patients of LSES $(p=0.48)$. Finally with respect to mortality, outcomes were not significantly improving for all races combined ( $p=0.41$ for effect of year), or when compared between non-Caucasian patients and Caucasian patients $(p=0.27)$, African-American patients and Caucasian patients $(p=0.07)$, Hispanic patients and Caucasian patients $(p=0.59)$, and lower and higher socioeconomic status patients $(p=0.19)$.

\section{Discussion}

As the US healthcare system continues to strive for highquality equal access treatment of all individuals, monitoring intervention rates as a function of race and socioeconomic status is critical. Not only does it provide a benchmark for current practices, but also identifies gaps in our widely varied healthcare landscape. The NIS appears to represent a key dataset with increasing utility in reporting trended patient interventions and outcomes on the basis of race and socioeconomic status. Further, the NIS is helpful in assessing pertinent associations between operative intervention and disposition for potentially susceptible demographic groups.

Data from the NIS database demonstrated that African Americans and LSES individuals suffering from TCSCIF had both lower odds of undergoing operative intervention and higher odds of unfavorable discharge disposition than their counterparts. In our multivariate model that controlled for race and socioeconomic status, race (but not socioeconomic status) was a factor in receiving surgery and both race and LSES impacted favorable discharge. There are 

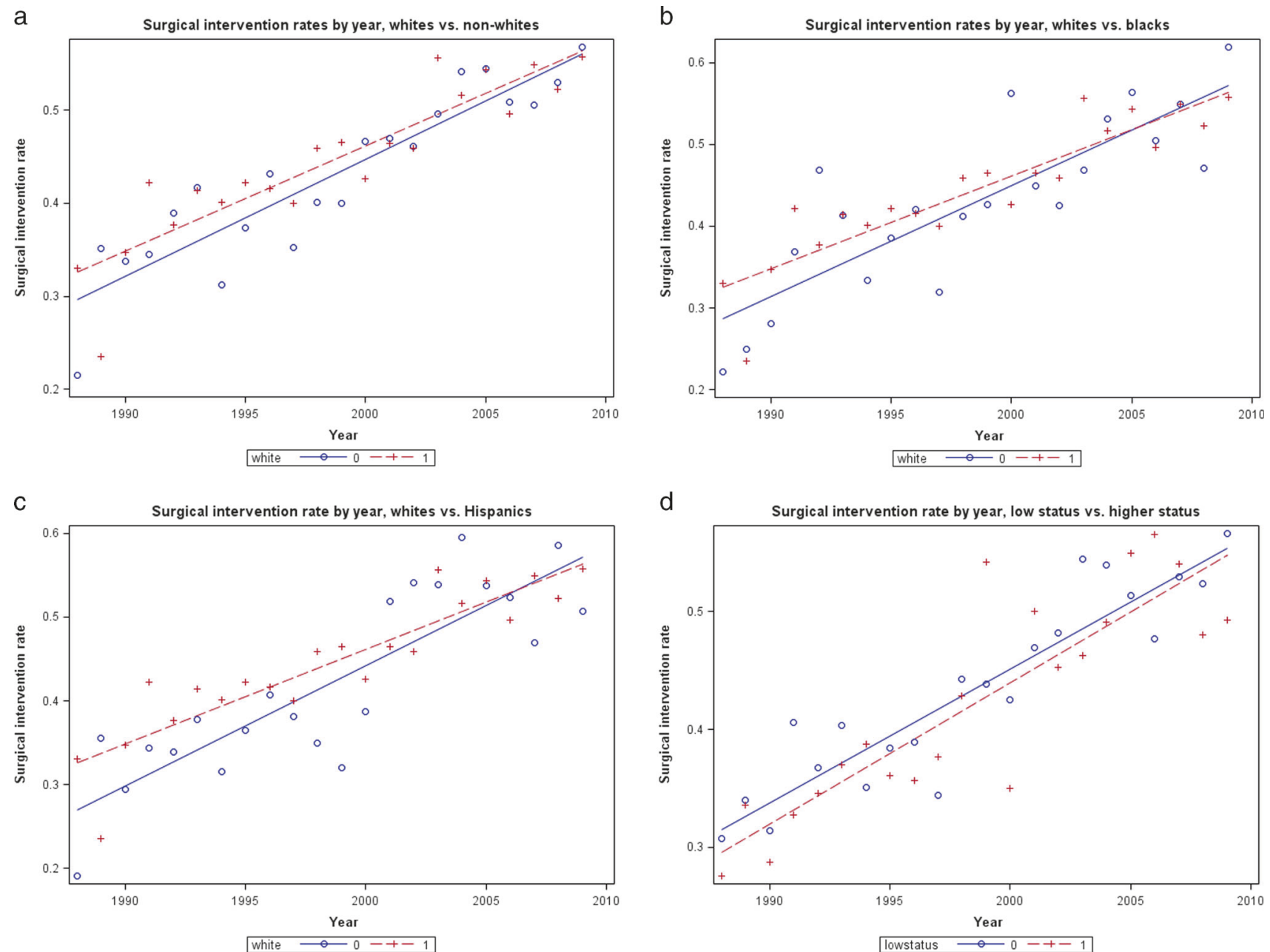

Fig. 1 Trends in surgical intervention rate from 1988-2009 for Caucasian patients and all non-Caucasian patients (graph a), AfricanAmerican patients (graph b), Hispanic patients (graph c), and high

multiple factors that could contribute to these observed differences. First, African Americans had greater odds of higher NISS than Caucasians. This raises the question of whether potentially other serious traumatic injuries within these populations negatively influence the possibility of undergoing surgical treatment. The etiology of traumatic spinal cord injury (TSCI) does exhibit variation between race. The third most common cause of TSCI in patients under 45 years old is gunshot wounds and accounts for $33 \%$ of injuries in African Americans, $14.6 \%$ in Hispanics, and only $3.0 \%$ in Caucasians from pooled data from the National Spinal Cord Injury Database and National Shriners Spinal Cord Injury Database [7, 18]. Future work to assess potential racial and/or socioeconomic differences in the etiology of TCSCIF merits further study.

Secondly, cultural differences with respect to trust in healthcare systems may influence patient and family decisions to seek intervention. This has been linked to lower rates of satisfaction with physicians and weakened

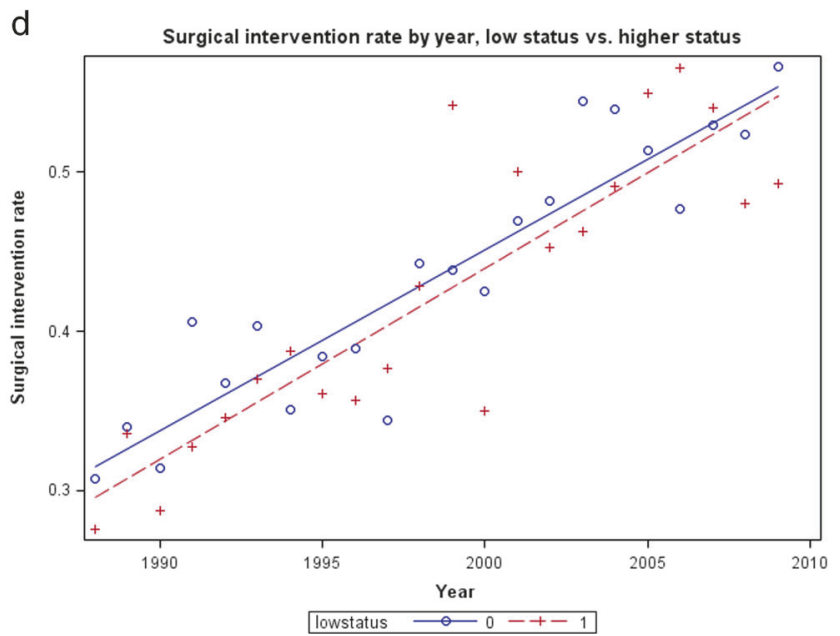

versus LSES (graph d). Note, Caucasian patients and higher socioeconomic status are represented by the red line, other races and LSES by the blue line

therapeutic alliance [19-23]. Multiple studies have documented, in both African American and Hispanic populations, an increased sense of physician distrust, concern for privacy, and worry of a potentially harmful experience in the hospital [24-26]. Finally, decreased access to healthcare in African American, Hispanic, and LSES individuals is a well-documented trend even within the last 5 years [27]. These factors, both individually and as a whole, may contribute to the decreased surgical intervention rates in at-risk populations.

The trends for operative intervention, and favorable discharge disposition for TCSCIF, are improving for nonCaucasian, African American, Hispanic, and low-income individuals over the study period. By 2009, surgical intervention rates had crossed (representing increased operative intervention rates than Caucasians) or consistently surpassed their benchmark comparison category, for at-risk races and socioeconomic levels, respectively. Favorable discharge rates were found to improve across the board as 

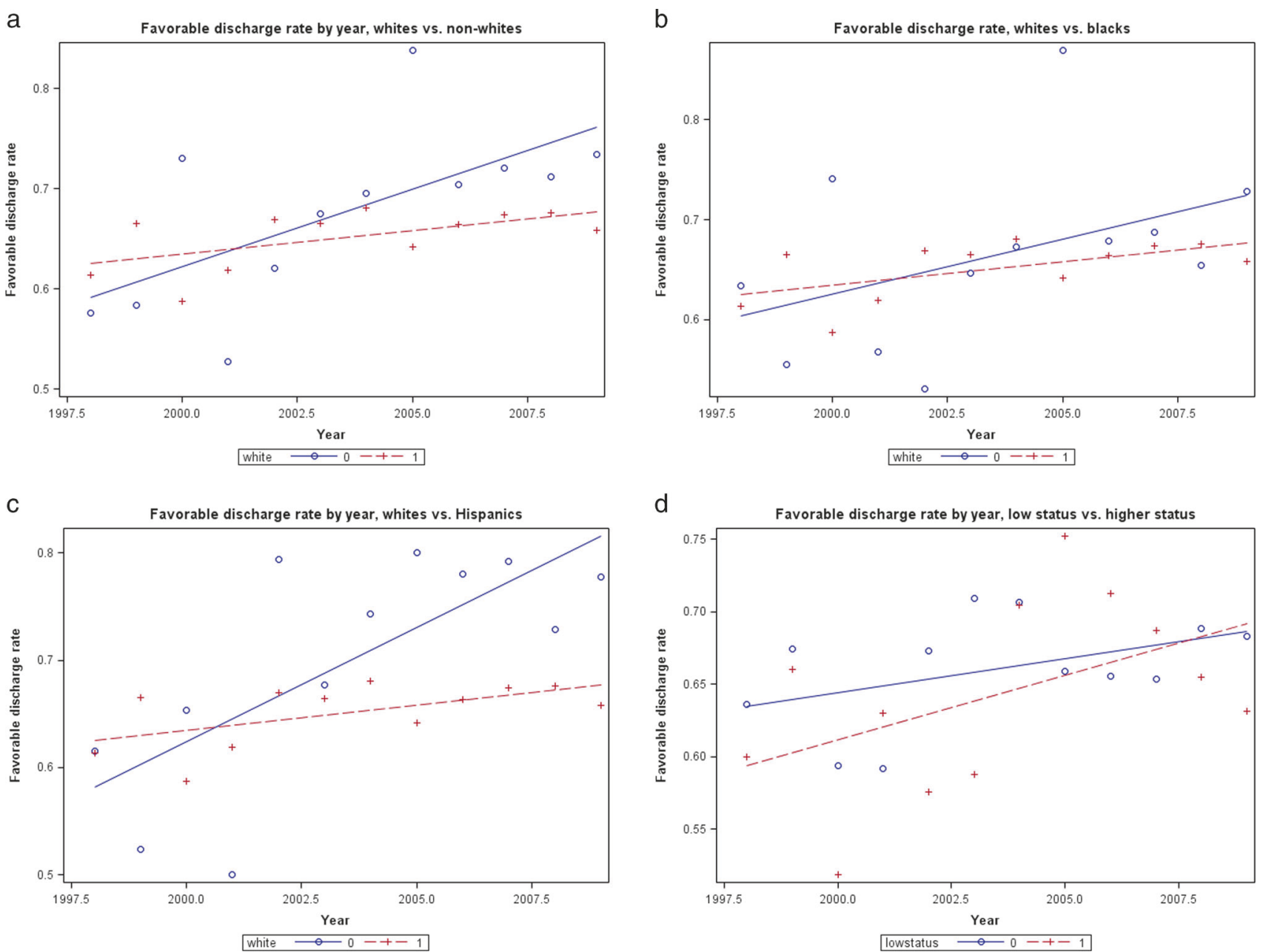

Fig. 2 Trends in favorable discharge rate from 1998-2009 for Caucasian patients and all non-Caucasian patients (graph a), AfricanAmerican patients (graph b), and Hispanic patients (graph c), and high

well. The rate of favorable discharges for Hispanic patients was outpacing its Caucasian benchmark. Mortality rates over the same time period observed were not statistically different between at-risk groups and their Caucasian or high socioeconomic counterpart.

Limitations of this study include its retrospective nature and the inherent potential for selection bias. The NIS does not provide clinical data with regard to certain patient factors and outcomes that may offer greater insight such as fracture type, neurologic and functional outcome, intensive care unit length of stay, or specific presenting neurological status. Secondly, we were unable to assess timing of surgical intervention, which could have significant implications for neurological recovery and discharge status. Thirdly, in performing the multivariate analysis combining race and socioeconomic status, $36 \%$ of the surgical intervention observations and $58 \%$ of the discharge observations were lost due to a lack of combined race/socioeconomic data. Finally, the relationship of NISS to overall clinical

versus LSES (graph d). Note, Caucasian patients and LSES are represented by the red line, other races and higher socioeconomic status by the blue line

status is unclear, therefore one cannot deduce the relationship of a higher NISS (with all racial groups assessed being higher than Caucasians) with the likelihood of surgical intervention, or favorable outcome.

The current US healthcare system is charged with providing equal access and quality care, irrespective of socioeconomic or racial factors. In the setting of TCSCIF, optimal implementation of appropriate surgical and medical management has been demonstrated to improve outcome, and therefore potential socioeconomic and racial disparities deserve further inquiry. This data may not only service as a benchmark for future comparison, but also encourage further investigation to underlying etiologies for race and socioeconomic disparities in care.

\section{Data archiving}

The data that supports the findings of this study are available from the NIS but restrictions apply to the availability of 
these data, which were used under license for the current study, and so are not publically available. Data are however available from the authors upon reasonable request and with permission of the NIS.

\section{Supplementary material}

The University of Florida Biostatistical Consulting Laboratory performed analyses based on data from the NISdatabase. Report 1 includes raw hospital demographics tables. Reports 2 and 3 include multivariate analyses of TCSCIF patients of different socioeconomic status and race with respect to surgical intervention rates, discharge, and mortality. Report 4 includes the multivariate analyses of TCSCIF patients with race and economic status as covariates with respect to surgical intervention rates, discharge, and mortality.

Acknowledgements This work was not funded by the NIH, or any other funding source.

Funding The authors declare no sources of funding for this study.

Author contributions ABD was the primary author of the article and responsible for amassing finished data summarising and compiling the completed manuscript. BR is the coprincipal investigator of the project, providing founding idea, and protocol for data discovery. He provided feedback on the report. DN contributed to extracting and analyzing NIS data, interpreting results, and creating summary tables and figures. SV contributed to interpretation of results and writing the article. DTL contributed to interpretation of results and writing the article. WCF contributed to interpretation of results and writing the article. BH contributed to the acquisition and interpretation of data and results for the article. DJH is the coprincipal investigator of the project, providing founding idea, and protocol for data discovery. He provided feedback on the report and was responsible for final proofing.

\section{Compliance with ethical standards}

Conflict of interest The authors declare that they have no conflict of interest.

Statement of Ethics We certify that all applicable institutional and governmental regulations concerning the ethical use of clinical data were followed during the course of this research. This was in adherence with the University of Florida Institutional Review Board (IRB\# 6272011).

Publisher's note: Springer Nature remains neutral with regard to jurisdictional claims in published maps and institutional affiliations.

\section{References}

1. Agency for Healthcare Research and Quality. National Healthcare Disparities Report. Rockville, MD: Agency for Healthcare Research and Quality; 2003.

2. Nelson A. Unequal treatment: confronting racial and ethnic disparities in health care. J Natl Med Assoc. 2002;94:666-8.
3. Kimball MM, Neal D, Waters MF, Hoh BL. Race and income disparity in ischemic stroke care: nationwide inpatient sample database, 2002 to 2008. J Stroke Cerebrovasc Dis. 2014;23:17-24.

4. Singh A, Tetreault L, Kalsi-Ryan S, Nouri A, Fehlings MG. Global prevalence and incidence of traumatic spinal cord injury. Clin Epidemiol. 2014;6:309-31.

5. DeVivo MJ, Go BK, Jackson AB. Overview of the national spinal cord injury statistical center database. J Spinal Cord Med. 2002;25:335-8.

6. DeVivo MJ. Epidemiology of spinal cord injury. In: Lin VW (Editor) Spinal cord medicine principles and practice New York: Demos Medical Publishing; 2010. p. 78-84.

7. Chen Y, Tang Y, Vogel LC, Devivo MJ. Causes of spinal cord injury. Top Spinal Cord Inj Rehabil. 2013;19:1-8.

8. Cripps RA, Lee BB, Wing P, Weerts E, Mackay J, Brown D. A global map for traumatic spinal cord injury epidemiology: towards a living data repository for injury prevention. Spinal Cord. 2011;49:493-501.

9. Furlan JC, Noonan V, Cadotte DW, Fehlings MG. Timing of decompressive surgery of spinal cord after traumatic spinal cord injury: an evidence-based examination of pre-clinical and clinical studies. J Neurotrauma. 2011;28:1371-99.

10. Carlson GD, Minato Y, Okada A, Gorden CD, Warden KE, Barbeau JM, et al. Early time-dependent decompression for spinal cord injury: vascular mechanisms of recovery. J Neurotrauma. 1997;14:951-62.

11. Dimar JR, Glassman SD, Raque GH, Zhang YP, Shields CB. The influence of spinal canal narrowing and timing of decompression on neurologic recovery after spinal cord contusion in a rat model. Spine. 1999;24:1623-33.

12. Fehlings MG, Rabin D, Sears W, Cadotte DW, Aarabi B. Current practice in the timing of surgical intervention in spinal cord injury. Spine. 2010;35(21 Suppl):S166-73.

13. Fehlings MG, Vaccaro A, Wilson JR, Singh A, W Cadotte D, Harrop JS, et al. Early versus delayed decompression for traumatic cervical spinal cord injury: results of the Surgical Timing in Acute Spinal Cord Injury Study (STASCIS). PLoS One. 2012;7:e32037.

14. Burke JF, Yue JK, Ngwenya LB, Winkler EA, Talbott JF, Pan JZ, et al. Ultra-early $(<12 \mathrm{~h})$ surgery correlates with higher rate of American Spinal Injury Association Impairment Scale conversion after cervical spinal cord injury. Neurosurgery. 2018. https://doi. org/10.1093/neuros/nyy537. [Epub ahead of print]

15. Lee DY, Park YJ, Kim HJ, Ahn HS, Hwang SC, Kim DH. Early surgical decompression within $8 \mathrm{~h}$ for traumatic spinal cord injury: is it beneficial? A meta-analysis. Acta Orthop Traumatol Turc. 2018;52:101-8.

16. Wilson JR, Tetreault LA, Kwon BK, Arnold PM, Mroz TE, Shaffrey $\mathrm{C}$, et al. Timing of decompression in patients with acute spinal cord injury: a systematic review. Glob Spine J. 2017;7(3 Suppl):95S-115S.

17. Anderson KK, Tetreault L, Shamji MF, Singh A, Vukas RR, Harrop JS, et al. Optimal timing of surgical decompression for acute traumatic central cord syndrome: a systematic review of the literature. Neurosurgery. 2015;77(Suppl 4):S15-32.

18. Devivo MJ. Epidemiology of traumatic spinal cord injury: trends and future implications. Spinal Cord. 2012;50:365-72.

19. Gamble VN. Under the shadow of Tuskegee: African Americans and health care. Am J Public Health. 1997;87:1773-8.

20. Gamble VN. A legacy of distrust: African Americans and medical research. Am J Prev Med. 1993;9:35-8.

21. Corbie-Smith G, Thomas SB, Williams MV, Moody- Ayers S. Attitudes and beliefs of African Americans to- ward participation in medical research. J Gen Intern Med. 1999;14:537-46.

22. Ayanian JZ, Cleary PD, Weissman JS, Epstein AM. The effect of patients' preferences on racial differences in access to renal transplantation. N Engl J Med. 1999;341:1661-9. 
23. Doescher MP, Saver BG, Franks P, Fiscella K. Racial and ethnic disparities in perceptions of physician style and trust. Arch Fam Med. 2000;9:1156-63.

24. Boulware LE, Cooper LA, Ratner LE, Laveist TA, Powe NR. Race and trust in the health care system. Public Health Rep. 2003;118:358-65.

25. Cooper-Patrick L, Gallo JJ, Gonzales JJ, Vu HT, Powe NR, Nelson C, et al. Race, gender, and partnership in the patientphysician relationship. JAMA. 1999;282:583-9.
26. Blendon RJ, Scheck AC, Donelan K, Hill CA, Smith M, Beatrice $\mathrm{D}$, et al. How white and African Americans view their health and social problems: different experiences, different expectations. JAMA. 1995;273:341-6.

27. Agency for Healthcare Research and Quality. Access and disparities in access to health care. (Rockville, MD). Content last reviewed June 2018. http://www.ahrq.gov/research/findings/ nhqrdr/nhqdr16/access.html. 